\title{
Comparative Performance Analysis of Soliton Pulse Generation Techniques
}

\author{
Devan Bhalla \\ ECE Dept., B.V.C.O.E. \\ New Delhi
}

\author{
Monica Bhutani \\ ECE Dept., B.V.C.O.E. \\ New Delhi
}

\begin{abstract}
Solitons are solitary waves which have a particle like behavior as they maintain their shape when travelling at a constant speed and also that when a soliton interacts with another soliton then it retains its shape after emerging from the collision with a possible change in the phase shift[1]. A precise simulation is employed to simulate the generation of soliton pulses by using two techniques employing the Laser Pulse as a source as well as by using a Soliton Pulse generator. The respective techniques performance in terms of bit rate, optical fiber length, Q factor, BER, jitter and eye opening were studied. The outcomes from the simulations further clarify that the Soliton Pulse generation technique provides much effective pulse shape retention for longer communication distances as well as provide a better peak power, both of which are crucial factors for the data transmission to be done efficiently in any communication system so as to compensate for the noise and the distortion introduced in the system. Also the non-linear effect of Self Phase Modulation has
\end{abstract}

\section{General Terms}

Soliton generation, Dispersion

\section{Keywords}

Bit rate, Q factor, SPM, Dispersion, jitter

\section{INTRODUCTION}

Solitons have been a topic of research both theoretically as well as experimentally[4] ever since their first observation was recorded by James Scott Russel in the year 1834 when he had observed a smooth shaped heap of water in a narrow canal which propagated for a few kilometers down the canal without any apparent change in its shape. However it was in the 1960's that a mathematical model was conceived along with a corresponding solution of the non-linear equation through an inverse scattering method. Though the concept of soliton was optics based it is equally important and applicable in other areas of science like in biology, communication networks, plasma and hydrodynamics to name a few. In optics, solitons are equally important and are generally classified based on the confinement of light in space or time. The solitary wave in the former case is referred to as spatial soliton and the latter is referred to as temporal soliton[9]. A third class of spatiotemporal solitons has also been observed recently. The soliton forming phenomenon arises from the non-linear properties of the medium through which a wave is propagating. In fiber optics in concern with the soliton generation, the Kerr Effect of Self Phase Modulation[3] is of importance as it balances out with the linear effect of dispersion for soliton pulse generation. In the case of spatial soliton the natural property of light which is to disperse in space is being compensated by the nonlinearity of the medium in such a way that higher intensity part of an optical beam which is typically at the center of the employed Gaussian beam, increase a value of refractive index of medium forming de facto a core of waveguide that is responsible to confine in reverse a dispersed light to the middle of the beam itself[5]. It can be easily intuitively understood that if the self induced nonlinearity[2] is too high the beam will get focused and on the other hand if it is very small or none, beam will disperse in space - a prevailing situation in many cases where a beam does not have enough power density to induce nonlinearity in a medium.

\section{MATHEMATICAL MODEL OF A SOLITARY WAVE}

A soliton is essentially a solitary wave which arises from the balanced of the non-linear and dispersive effects[6]. The propagation of light can be precisely described mathematically with Maxwell equations. When equations for magnetic and electric fields are combined together[7]:

$\nabla^{2} \mathrm{E}-\left(1 / \mathrm{c}^{2}\right) \partial^{2} \mathrm{E} / \partial \mathrm{t}^{2}=\left(1 / \varepsilon_{0} \mathrm{c}^{2}\right) \partial^{2} \mathrm{P} / \partial \mathrm{t}^{2}$

-Eq.(1).

where $c$ is the speed of light in the vacuum and $\varepsilon_{0}$ is the vacuum permittivity. The induced polarization $\mathrm{P}$ consists of two parts such that:

$\mathrm{P}(\mathrm{r}, \mathrm{t})=\mathrm{P}_{\mathrm{L}}(\mathrm{r}, \mathrm{t})+\mathrm{P}_{\mathrm{NL}}(\mathrm{r}, \mathrm{t})$

where the linear part $P_{L}$ and nonlinear part $P_{N L}$ are related to the electric filed by the general relations:

$$
\begin{aligned}
& \mathrm{PL}(\mathrm{r}, \mathrm{t})=\varepsilon_{0} \int \chi^{(1)}\left(\mathrm{t}-\mathrm{t}^{\prime}\right) \cdot \mathrm{E}(\mathrm{r}, \mathrm{t}) \mathrm{dt} \\
& \mathrm{P}_{\mathrm{NL}}(\mathrm{r}, \mathrm{t})=\varepsilon_{0} \int \chi^{(3)}\left(\mathrm{t}-\mathrm{t}_{1} \cdot \mathrm{t}-\mathrm{t}_{2}, \mathrm{t}-\mathrm{t}_{3}\right) \times \mathrm{E}\left(\mathrm{r}, \mathrm{t}_{1}\right) \mathrm{E}\left(\mathrm{r}, \mathrm{t}_{2}\right) \mathrm{E}\left(\mathrm{r}, \mathrm{t}_{3}\right) \mathrm{dt}_{1} \mathrm{dt}_{2} \mathrm{dt}_{3} \\
& \text {-Eq.(4). }
\end{aligned}
$$

where $x^{(1)}$ and $\chi^{(3)}$ are the first- and third- order susceptibility tensors and limits extend from -infinity to infinity.

\section{SOLITON GENERATION AND RESULT ANALYSIS}

(i). Using Laser as Source-

In the given Setup of Fig.1. the Soliton pulses are generated by applying an RZ Soliton shape electrical driver to a linear amplitude modulator, which transmits a Continuous Wave $(\mathrm{CW})$ laser source which is centered at $1550 \mathrm{~nm}$.

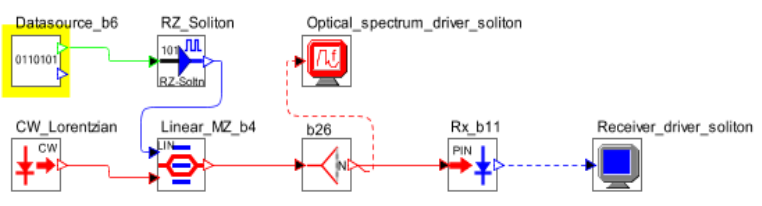

Fig.1. Simulation setup 
a. Bit rate variation- A parametric scan was applied and the output was noted on the spectrum analyzer.

\begin{tabular}{l|l} 
Run \# & Param 1 \\
\hline Parameter & Bit_rate \\
\hline Run 1 & 1 \\
\hline Run 2 & 5 \\
\hline Run 3 & 10 \\
\hline Run 4 & 20 \\
\hline Run 5 & 50 \\
\hline Run 6 & 100
\end{tabular}

Fig.2. Parametric Scan on Bit rate

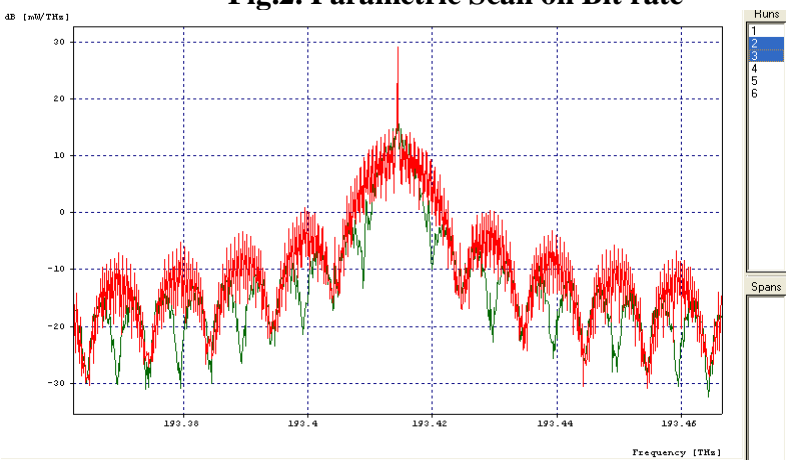

Fig.3. Output Spectrum Analyzer

It was observed that with the Laser source being utilized, as the bit rate increases the non-linearity gets introduced and the pulse begins to spread more leading to an increase in dispersion with the increase in bit rate.

b. Variation in the length of the optical fiber- The length of the optical fiber link is a crucial parameter as it is limited at higher distances due to increasing values of dispersion which lead to the distortion of the data transmitted. A parametric scan was applied on the length and the eye diagrams were observed. Initially the parametric scan was applied from 1$1000 \mathrm{~km}$ but based on the minimum eye diagram distortion in the particular range of operation the operational range was reduced to up to $200 \mathrm{~km}$.

\begin{tabular}{l|l|}
\hline Run \# & Param 1 \\
\hline 2arameter & length \\
\hline Run 1 & 100.0 \\
\hline Run 2 & 125.0 \\
\hline Run 3 & 150.0 \\
\hline Run 4 & 175.0 \\
\hline Run 5 & 200.0 \\
\hline
\end{tabular}

Fig.4. Parametric Scan on Length of the optical fiber

Now as the Length of the Optical Fiber Link begins to increase, the amplitude of the Eye Diagram of the corresponding Length of the Optical fiber link begins to decrease rapidly with each successive run as is represented as in Fig.3.5.



Fig.5. Eye Diagram

(ii). Using Soliton Pulse Generator-

In the following setup as shown in Fig.3.6., the soliton pulses are generated by using as optical source a soliton pulse generator externally modulated by a linear amplitude modulator, which is driven by a NRZ signal.

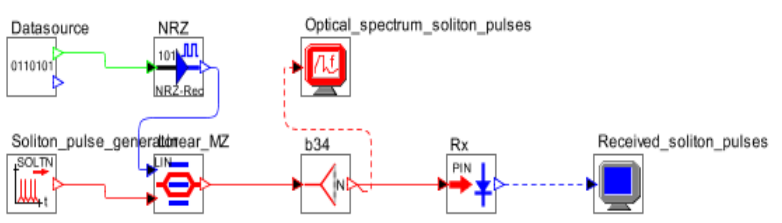

Fig.6. Simulation Setup

a. Bit rate variation- A parametric scan was applied on the bit rate as in Fig.3.2. and the spectrum analyzers outputs were superimposed through the ImportExport feature of the OptSim software and it was comprehend that the shape of the soliton pulse remains the same throughout even while varying the bit rate. Thus making the Soliton Pulse generator efficient as compared to the Laser Pulse Source.

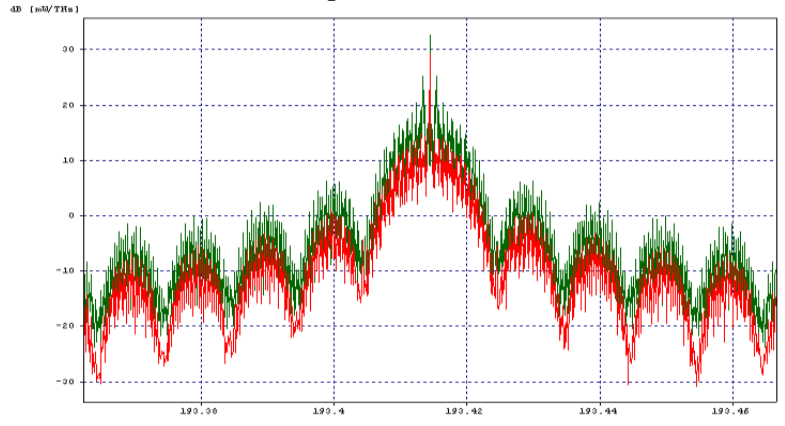

Fig.7.

Through the Eye diagrams it was observed that the Laser Pulse source has a lower sensitivity as its rate of eye closure is smaller whereas the Soliton Pulse generator offers higher sensitivity as its rate of eye closure is larger. Also the height of the eye diagram which dictates the ideal time to receive and sample the waveform, is measured when the height of the eye opening is largest clearly shows that the Laser pulse provides shorter height and thus has a higher noise level whereas the Soliton pulse generator provides a larger height and thus works well at higher bit rates without affecting the soliton pulse shape thus providing an efficient mechanism to transfer data in an optical communication system. The width of the 
eye opening gives a measure of the effective time interval over which the received signal can be sampled without error which is evidently larger in the case of a Soliton Pulse generator thus providing a larger time interval to sample without error contrarily as in the Laser pulse technique



Fig.3.8. Eye Diagram.

a. Variation in the length of the optical fiber- A parametric scan was applied on the length and the eye diagrams were observed. Initially the Parametric Scan was implemented from 100 $\mathrm{km}$ up to $500 \mathrm{~km}$ and the region of operation was narrowed down to $150 \mathrm{~km}-275 \mathrm{~km}$ based on the observation from the Eye Diagrams corresponding to the particular Optical fiber lengths. The Eye Diagrams at $150 \mathrm{~km}, 200 \mathrm{~km}$, $250 \mathrm{~km}$ up to $500 \mathrm{~km}$ were studied so as to narrow down to the desired region of operation so as to counter the distortion in the Eye Diagram. In the Following section the Eye Diagrams between the Optical Fiber Lengths of 150-350 $\mathrm{km}$ were studied since the Eye diagram began to produce distortions. Hence the region of operation was reduced to be centered around 150 to $275 \mathrm{~km}$ on which a new Parametric scan was then applied upon.

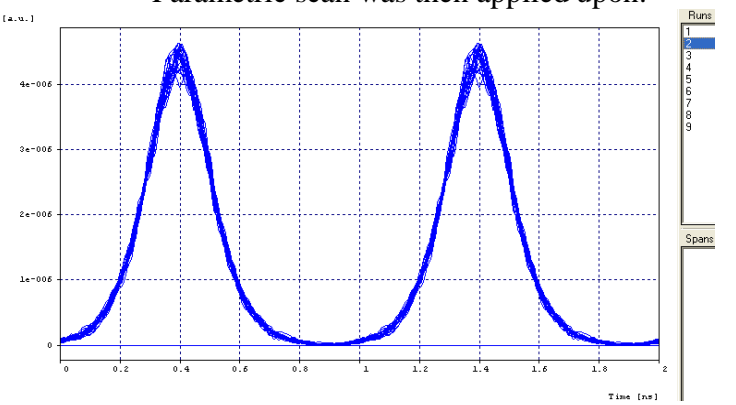

Fig.9.(a). at $150 \mathrm{~km}$

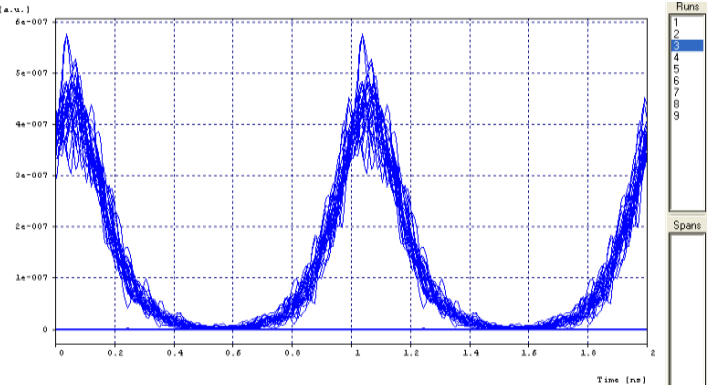

Fig.9.(b). at $200 \mathrm{~km}$

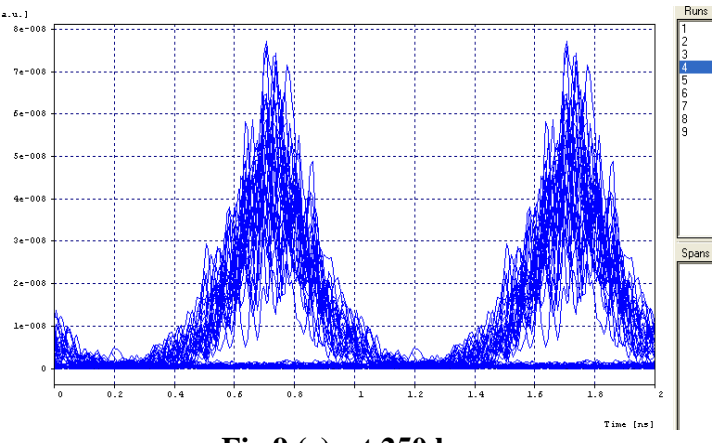

Fig.9.(c). at 250 km

Now based on the distortion introduced in the eye diagrams at the peaks the operational range of length of the fiber was further reduced to $150-275 \mathrm{~km}$.

\begin{tabular}{|l|l|}
\hline \multicolumn{1}{|l|}{ Run \# } & Param 1 \\
\hline Parameter & Length \\
\hline Run 1 & 150.0 \\
\hline Run 2 & 175.0 \\
\hline Run 3 & 200.0 \\
\hline Run 4 & 225.0 \\
\hline Run 5 & 250.0 \\
\hline Run 6 & 275.0
\end{tabular}

Fig.10. Parametric Scan on the Length of the optical fiber

The following are the Eye Diagrams of the Signal at an Optical Fiber link length of 150km (Fig.11.(a).) and $175 \mathrm{~km}$ (Fig.11.(b).) respectively. The latter eye diagram shows the distortion being introduced at the peaks.



Fig11.(a). at $150 \mathrm{~km}$ 


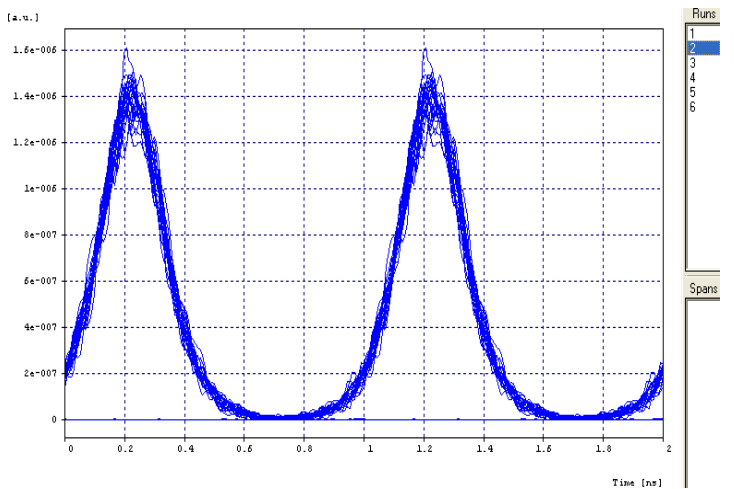

Fig.11.(b). at 175 km

Also, as the Length of the Optical Fiber Link begins to increase, the amplitude of the Eye Diagram of the corresponding Length of the Optical fiber link begins to decrease rapidly[8] with each successive run as shown in Fig.12. but here the decrease in power is much less In the above Fig.12.., the Amplitude of the Eye diagram of Run \#3 at $200 \mathrm{~km}$ has the smallest amplitude (Green) when compared to the Eye Diagrams of Run's \#2 and \#1. Here Run \#2 corresponds at $175 \mathrm{~km}$ (Brown) and Run \#1 corresponds to the Eye Diagram at $150 \mathrm{~km}$ (Blue).

In comparison to the Laser Pulse generator, the Soliton Pulse generator offers much less decrease in the amplitudes of the heights of the eye diagrams with the corresponding increase in length thus indicating that it retains the peak power over longer distances of propagation.
Through the Electric Spectrum at a bit rate of $10 \mathrm{Gbps}$ it was confirmed that the Soliton Pulse generator provides a better peak power as compared to the Laser Pulse generator.

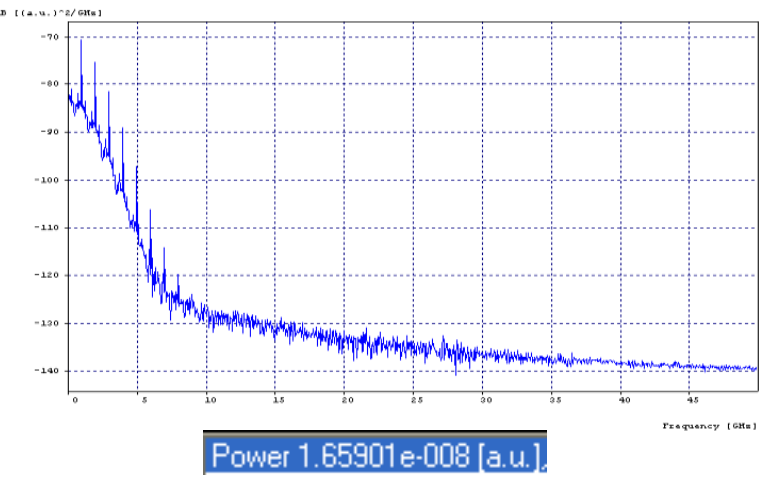

Fig.13.(a). Electric Scope of a Laser Pulse Generator

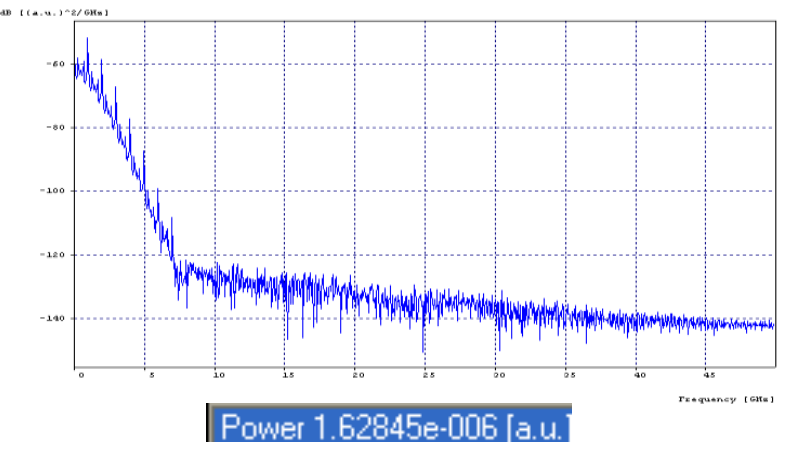

Fig.13.(b). Electric Scope of a Soliton Pulse Generator

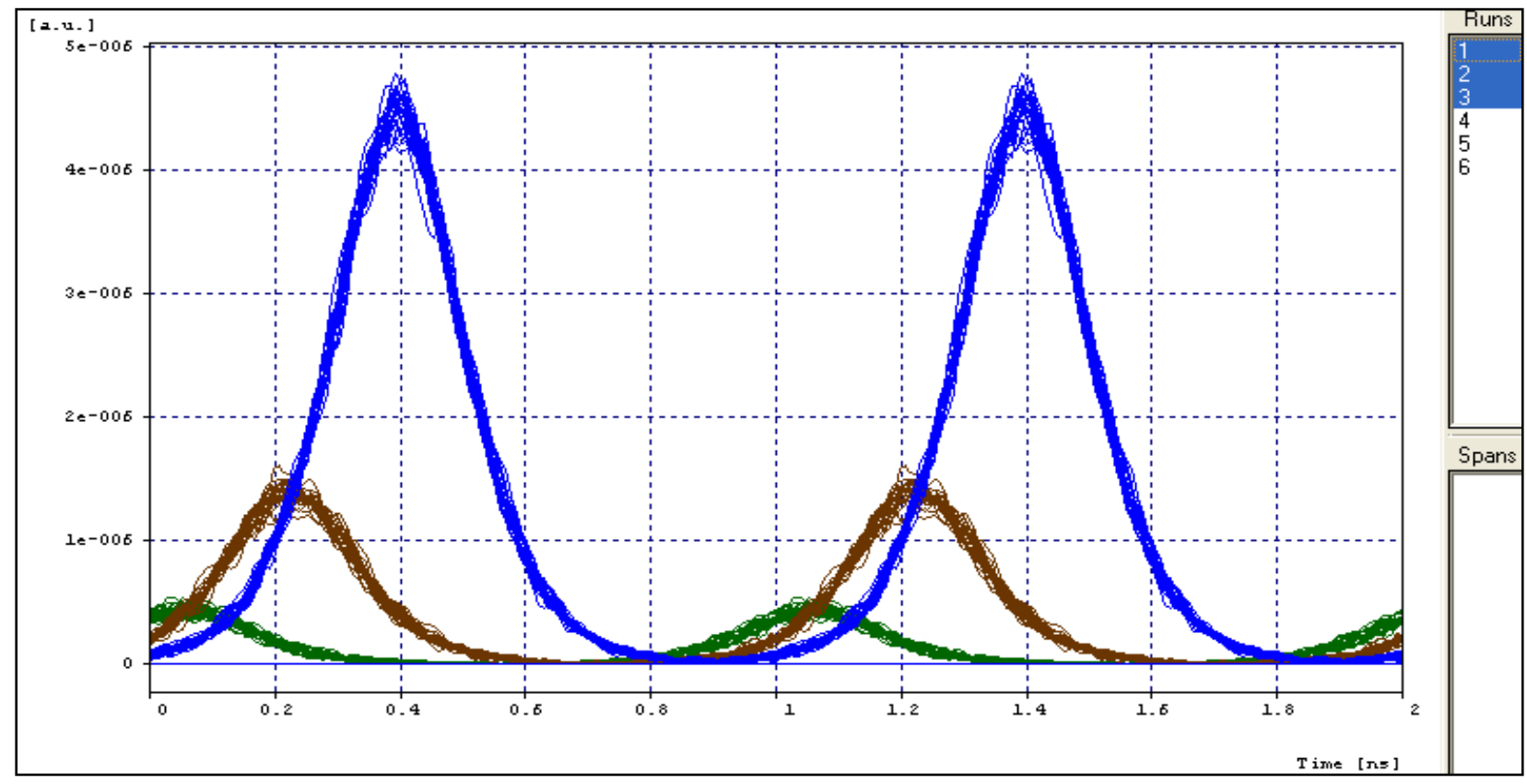

Fig.12. 
Table 1.

\begin{tabular}{|c|c|}
\hline Parameter & Assigned Values \\
\hline Length of optical fiber link & $150 \mathrm{~km}$ \\
\hline Bit rate & $10 \mathrm{Gbps}$ \\
\hline Frequency range of operation & $192-194 \mathrm{THz}$. \\
\hline
\end{tabular}

(iii). Q factor, BER, Timing Jitter and Eye opening when a Laser Pulse source is used: The important parameters of $\mathrm{Q}$ factor, BER, Jitter as well as that of eye opening were recorded at lengths of $1 \mathrm{~km}$ and $50 \mathrm{~km}$ respectively. The value of the Q factor and eye opening decreases whereas those of Jitter and BER (Bit Error Rate) increases as shown in Table 2.

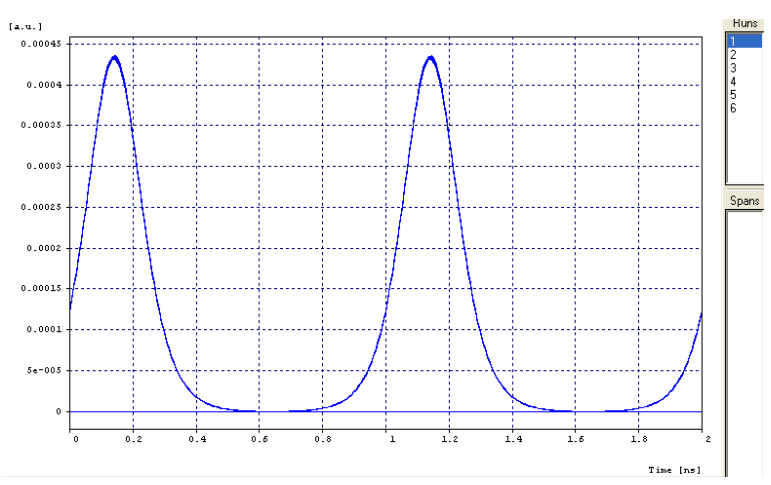

Fig.14.(a). at 1 km

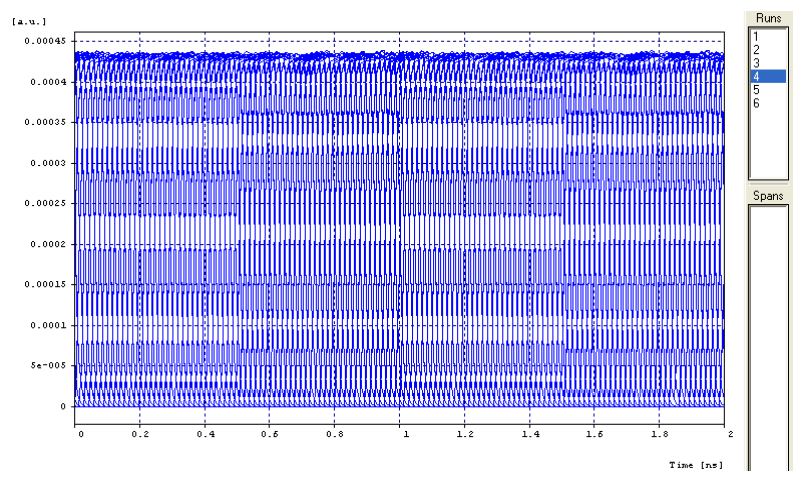

Fig.14.(b). at $50 \mathrm{~km}$

Table.2.

\begin{tabular}{|c|c|c|}
\hline \multirow{2}{*}{ Parameter } & \multicolumn{2}{|c|}{ Length of Optical fiber link } \\
\cline { 2 - 3 } & At $\mathbf{1 ~ k m}$ & At $\mathbf{5 0} \mathbf{~ k m}$ \\
\hline Q factor & $40.000 \mathrm{~dB}$ & $12.763 \mathrm{~dB}$ \\
\hline BER & $1 \mathrm{e}^{-40}$ & $6.936 \mathrm{e}^{-06}$ \\
\hline Eye Opening & 0.0043 a.u. & 0.00016 a.u. \\
\hline Jitter & $0.0038 \mathrm{~ns}$ & $0.1822 \mathrm{~ns}$ \\
\hline
\end{tabular}

The above parameters have been studied and their respective readings recorded with respect to the change in the optical fiber length. A similar set of readings have been recorded using the Soliton Pulse generator as the source.

(iv). Q factor, BER, Timing Jitter and Eye opening when a Soliton Pulse generator is used as the source: The important parameters of Q factor, BER, Jitter as well as that of eye opening were recorded at lengths of $150 \mathrm{~km}$ and $275 \mathrm{~km}$ respectively as with a soliton pulse generator the range of the optical fiber lengths were increased considerably since the soliton pulse can retain its shape for longer distances. The value of the $\mathrm{Q}$ factor and eye opening decreases whereas those of Jitter and BER increases as shown in Table 3.

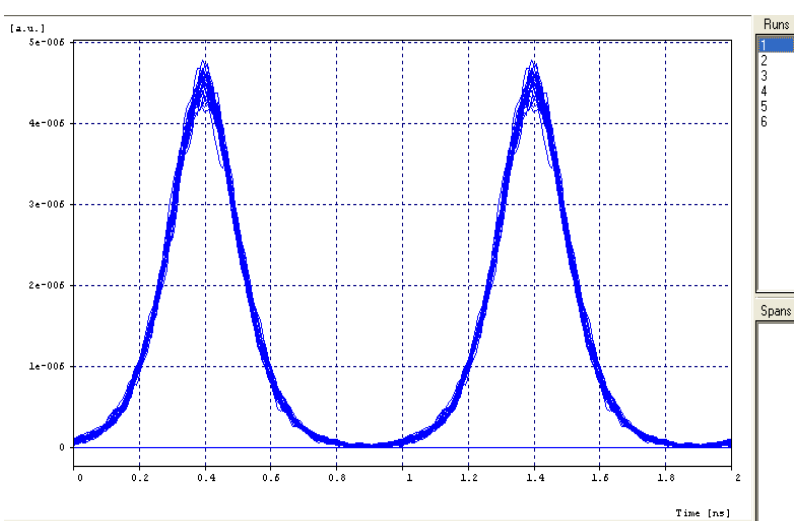

Fig.15.(a). at $150 \mathrm{~km}$

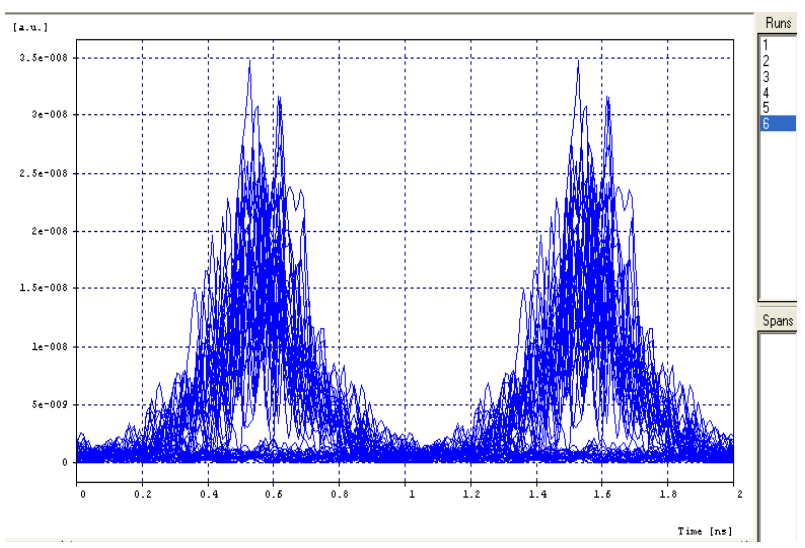

Fig.15.(b). at 275 km

Table.3.

\begin{tabular}{|c|c|c|}
\hline \multirow{2}{*}{ Parameter } & \multicolumn{2}{|c|}{ Length of Optical fiber link } \\
\cline { 2 - 3 } & At 150 km & At 275 km \\
\hline Q factor & $32.539 \mathrm{~dB}$ & $9.384 \mathrm{~dB}$ \\
\hline BER & $1 \mathrm{e}^{-40}$ & $5.374 \mathrm{e}^{-11}$ \\
\hline Eye Opening & $4.07956 \mathrm{e}^{-06}$ & $8.5971 \mathrm{e}^{-07}$ \\
\hline Jitter & $0.0117 \mathrm{~ns}$ & $0.0328 \mathrm{~ns}$ \\
\hline
\end{tabular}

From Table.2. and Table.3. it is clarified that the performance of the Soliton Pulse Generator is superior to that of the Laser 
Pulse source as it provides an efficient Q factor value, a lower BER at higher propagation distances of the optical fiber length, a comparatively better eye opening and a considerably improved value of Jitter. All the above parameters are very crucial in the performance analysis of any communication system.

\section{CONCLUSIONS AND FUTURE SCOPE}

In this paper the work has been reported on the Soliton generation methods using Laser source as well as using a Soliton Pulse Generator and their performance analysis has been done by varying the bit rate as well as the length of the optical fiber link. Here, it has been demonstrated that the effect of utilizing the Soliton Pulse generator results in better and improved values of the crucial parameters of $Q$ factor, BER, Jitter and eye opening. The Block Mode of the software has not been studied and can be undertaken as the future area of study under which individual blocks can be modeled and the effects of various crucial parameters can be studied and their effect on the performance of the optical communication system can be analyzed. Also, the mathematical modeling of the soliton wave using the Korteweg-de Vries equation (KdV equation) needs to be addressed in the future work to understand the behavior of the solitons in a better way. The Polarization effects of the solitons have to be studied and their analysis needs to be undertaken to promote a better understanding of the solitons.

\section{REFERENCES}

[1] M.-C. Chan et al., "1.2- to 2.2- $\mu$ m tunable Raman soliton source based on a Cr:forsterite laser and a photoniccrystal fiber", IEEE Photon. Technol. Lett. 20 (11), 900 (2008)

[2] J. H. Lee et al., "Soliton self-frequency shift: experimental demonstrations and applications", IEEE J. Quantum Electron. 14 (3), 713 (2008)

[3] J. Toulouse, 'Optical nonlinearities in fibers: review, recent examples, and system applications'. Light wave Technol., 23(11), pp. 3625-3641, 2005

[4] PORSEZIAN, K., KURIAKOSE, V.C. Optical Solitons : Theoretical and Experimental challenges. 1st edition. Berlin : Springer, 2003. 406 s. ISBN 3-540-00155-7.

[5] Santhanam J., Agrawal GP, Raman-induced spectral shifts in optical fiber: general theory based on the moment method, Opt. Commun. 222,413(2003)

[6] AGRAWAL, Govind P. Applications on Nonlinear Fiber Optics. San Diego: Academic Press, 2001. 458 s. ISBN 0-12-045144-1.

[7] V. N. Serkin and A. Hasegawa, "Novel soliton solutions of the nonlinear Schrödinger equation model", Phys. Rev. Lett. 85 (21), 4502 (2000)

[8] Kivshar, Yuri S., Agrswal, Govind P, Optical Solitons from fibers to Photonic crystals, San Diego; Academic Press, 2003.540s.ISBN 0-12-410590-4

[9] Gagliardi, Robert M, KARP, Sharman, Optical Communications, $2^{\text {nd }}$ edition New York; $1995.347 \mathrm{~s}$ ISBN 0-471-54287-3 